JGG 2020;68:152-158

doi: 10.36150/2499-6564-391

\section{Prognostic factors in older patients admitted in ICU with diagnosis of abdominal sepsis (sepsis-3 criteria)}

\author{
Francesca Remelli', Alberto Fogagnolo², Amedeo Zurlo', \\ Carlo Alberto Volta ${ }^{2}$, Savino Spadaro², Stefano Volpato ${ }^{1}$ \\ ${ }^{1}$ Geriatrics e Ortogeriatrics Unit, Department of Medical Sciences, University of Ferrara, \\ Italy; ${ }^{2}$ Anesthesiology and Resuscitation Unit, Department of Morfology, Surgery and Sperimental \\ Medicine, University of Ferrara, Italy
}

Background \& aims. In septic older patients, hospitalization in Intensive Care Unit (ICU) is often associated with unfavourable outcomes. The aim of this study was to estimate the prevalence of geriatric age in patients admitted to ICU for abdominal sepsis and to investigate predictors of mortality, including quickSOFA, a recent prognostic index proposed for septic patients.

Methods. Prospective observational study on patients admitted to ICU of St. Anna Hospital (Ferrara) between January and July 2016 with the following inclusion criteria: 1) adults (18 years); 2) abdominal infection treated with surgically techniques; 3) admission to ICU. Demographic, clinical data and survival at 30 and 90-day were collected for each patient.

Results. Thirty patients were enrolled, and the majority of geriatric patients $(80 \% \geq 65$ years and $53 \% \geq 75$ years). The 30 and 90 -day mortality was 17 and $37 \%$, respectively. Almost all deaths occurred in older age (mortality rate of $42 \%$ ). SOFA (Sepsis Related Organ Failure Assessment) score was the prognostic index most frequently associated with exitus at $30(A \cup C=0.702)$ and 90 days $(A \cup C=0.730)$. quickSOFA was only related to early mortality (AUC $=0.640$ ).

Conclusions. Our data confirm the high geriatric prevalence among patients admitted to ICU for abdominal sepsis, underlining the advanced age as a negative prognostic factor. SOFA score appears a reliable prognostic indicator of mortality, also in older population, while quickSOFA proved effective only for short-term mortality.

How to cite this article: Remelli $F_{1}$ Fogagnolo A, Zurlo A, et al. Prognostic factors in older patients admitted in ICU with diagnosis of abdominal sepsis (sepsis-3 criteria) Journal of Gerontology and Geriatrics 2020;68:152-8. https://doi. org/10.36150/2499-6564-391

C Copyright by Società Italiana di Gerontologia e Geriatria (SIGG)

(c) (1) (3) $(9)$

OPEN ACCESS

This is an open access article distributed in accordance with the CC-BY-NC-ND (Creative Commons Attribution-NonCommercial-NoDerivatives 4.0 International) license. The article can be used by giving appropriate credit and mentioning the license, but only for non-commercial purposes and only in the original version. For further information: https://creativecommons.org/licenses/by-nc-nd/4.0/deed.en
Key words: elderly, abdominal sepsis, intensive care, quickSOFA

\section{INTRODUCTION}

Sepsis and septic shock are major health problems: worldwide, millions of people are affected every year, with an overall mortality rate exceeding $25 \%$. Sepsis is also one of the first causes of death in older patients. The incidence of sepsis is growing, as a result of the increase in the mean population age, with consequent increase in comorbidities, and greater efficacy in diagnosis ${ }^{1,2}$. Age plays a key role as independent prognostic factor for morbidity and mortality in septic patients: early intra-hospital mortality is more frequent in elderly patients, while, among survivors, disability is more likely ${ }^{3}$. 
The diagnosis of sepsis is complex, due to the high variability of the clinical picture and the lack of pathognomonic signs or symptoms. To promote an early identification and a timely treatment, crucial for prognosis, in 2016, current definitions of sepsis and septic shock were modified by the Third International Consensus Definitions for Sepsis and Septic Shock (Sepsis-3) ${ }^{4,5}$. Sepsis was re-defined as a syndrome characterized by one or more life-threatening organ dysfunctions, due to an altered host's response to infection. Therefore, sepsis should be diagnosed from an acute increase of 2 or more SOFA points (compared to the patient's basic conditions). SOFA score is a system to assess the presence and the grade of organ failure, created on septic patients admitted to ICU ${ }^{6}$. An increase in SOFA points is correlated to a higher risk of mortality ${ }^{7}$. The SOFA score is calculated based on 6 physiological variables, that are index of the degree of a system's function (cardiocirculatory, respiratory, renal, hepatic, coagulative and nervous). Furthermore, each of these variables is given a score from 0 (normal) to 4 (seriously compromised), according to the criteria shown in Table I. The SOFA score can be re-calculated every 24 hours to monitor the patient's clinical evolution in ICU ${ }^{6}$.

On the other hand, septic shock is identified in a septic patient (SOFA $\geq 2$ ) who has persistent hypotension, despite of an adequate fluid therapy, which requires the administration of vasoactive amines, in order to maintain a Mean Arterial Pressure (MAP) $>65 \mathrm{mmHg}$, and a with serum lactate levels $>2 \mathrm{mmol} / \mathrm{L}$. Under these septic shock evaluation criteria, hospital mortality was reported to be higher than $40 \%{ }^{4}$. In addition, to better identify patients with the highest death risk from sepsis, a new prognostic score was introduced as a modified version of SOFA. This new score, called quickSOFA (qSOFA), it is expected to be simple and easily accessible to healthcare personnel, even outside the hospital, since it does not require any laboratory test. Specifically, three clinical parameters are evaluated: tachypnea (Respiratory Rate $\geq 22 /$ min), altered mental status (Glasgow Coma Scale $\leq 14$ ) and arterial hypotension (Systolic Arterial Pressure $\leq 100 \mathrm{mmHg}$ ). A binary score, $0 / 1$, is assigned to each one of these parameters, so that the maximum overall qSOFA score is 3. A qSOFA $\geq 2$ is associated with a high risk of mortality due to sepsis. Previous studies reported a higher risk of mortality of $8 \%$ in patients with qSOFA equal to 2 and greater than 20\% with qSOFA equal to $3^{4}$. However, the qSOFA still needs a prospective validation to confirm its real efficacy, as it was created on retrospective studies on acute patients, without any distinction by age.

Among the different origin sites of sepsis and septic shock, the intra-abdominal infection is the second most frequent cause in critically ill patients (20-25\% of total), after pulmonary infections. According to several studies carried out in an Intensive Care Setting ${ }^{8}, 51 \%$ of all patients admitted is infected and $19 \%$ of these have an intra-abdominal infection. Abdominal sepsis is a complication of intra-abdominal infection characterized by

Table I. Sequential [sepsis related] Organ Failure Assessment (SOFA) Score.

\begin{tabular}{|c|c|c|c|c|c|}
\hline & \multicolumn{5}{|c|}{ Score } \\
\hline & 0 & 1 & 2 & 3 & 4 \\
\hline \multicolumn{6}{|c|}{ Respiratory system } \\
\hline $\mathrm{PaO}_{2} / \mathrm{FiO}_{2}(\mathrm{mmHg})$ & $\geq 400$ & $<400$ & $<300$ & $\begin{array}{c}<200 \text { with respiratory } \\
\text { support }\end{array}$ & $\begin{array}{c}<100 \text { with respiratory } \\
\text { support }\end{array}$ \\
\hline \multicolumn{6}{|c|}{ Coagulation } \\
\hline Platelets $\times 10^{3} / \mu \mathrm{L}$ & $\geq 150$ & $<150$ & $<100$ & $<50$ & $<20$ \\
\hline \multicolumn{6}{|c|}{ Hepatic system } \\
\hline Bilirubin (mg/dL) & $<1.2$ & $1.2-1.9$ & $2.0-5.9$ & $6.0-11.9$ & $\geq 12.0$ \\
\hline \multicolumn{6}{|c|}{ Cardiovascular system } \\
\hline & $\begin{array}{l}\mathrm{MAP} \geq 70 \\
\mathrm{mmHg}\end{array}$ & $\begin{array}{l}\mathrm{MAP}<70 \\
\mathrm{mmHg}\end{array}$ & $\begin{array}{c}\text { Dopamine }<5 \text { or } \\
\text { Dobutamine (any dose) }\end{array}$ & $\begin{array}{c}\text { Dopamine } 5,1-15 \text { or } \\
\text { norepinephrine } \leq 0.1 \text { or } \\
\text { epinephrine } \leq 0.1^{\mathrm{a}}\end{array}$ & $\begin{array}{c}\text { Dopamine }>15 \text { or } \\
\text { norepinephrine }>0.1 \text { or } \\
\text { epinephrine }>0.1^{\mathrm{a}}\end{array}$ \\
\hline \multicolumn{6}{|c|}{ Central nervous system } \\
\hline Glasgow Coma Scale & 15 & $13-14$ & $10-12$ & $6-9$ & $<6$ \\
\hline \multicolumn{6}{|c|}{ Renal system } \\
\hline Creatinine $(\mathrm{mg} / \mathrm{dl})$ & $<1.2$ & $1.2-1.9$ & $2.0-3.4$ & $3.5-4.9$ & $>5.0$ \\
\hline Urine output (ml/day) & & & & $<500$ & $<200$ \\
\hline
\end{tabular}

Abbreviations: Pa02, partial pressure of oxygen; Fi0 2, fraction of inspired oxygen; MAP, mean arterial pressure.

Notes: a All catecholamines doses represent $\mu \mathrm{g} / \mathrm{kg} / \mathrm{min}$. 
the highest rate of deaths (30 to $60 \%$ ), as compared to those of other primitive sites ${ }^{9,10}$.

The literature currently available on this subject is extremely limited and there are no randomized controlled trials regarding the efficacy of qSOFA as prognostic score in older population affected by abdominal sepsis. In a recent prospective study performed on 272 older patients with sepsis, admitted in ICU, qSOFA seems to be ineffective to predict intra-hospital, while seems to be correlated to mortality three months after discharge ${ }^{11}$. Moreover, a retrospective cohort study, published in 2018 and performed in Finland, has shown an insufficient sensitivity of qSOFA as prognostic score in septic adult population with a diffuse secondary peritonitis, without any age distinction ${ }^{12}$.

Among the recent guidelines recommended for abdominal sepsis management, in addition to a surgical source control and an intensive support of organ function ${ }^{13}$, it is necessary to start timely an appropriate antimicrobial therapy at high doses, namely effective on the microorganisms that sustain the infection ${ }^{14,15}$.

The present study is aimed to analyze predictors of mortality for sepsis, in particular testing qSOFA as score for negative outcomes in older patients with abdominal sepsis admitted in ICU.

\section{MATERIALS AND METHODS}

This is a prospective observational study carried out in the Intensive Care Unit (ICU) of St. Anna Hospital (Ferrara), between January and July 2016. The study enrolled patients admitted to ICU with all the following inclusion criteria: 1 ) adults ( $\geq 18$ years); 2 ) presence of abdominal infection treated with percutaneous or surgically techniques; 3) admission to ICU for abdominal sepsis. The initial diagnosis of sepsis was carried out through the 2001 criteria $^{5}$; subsequently, these criteria were compared with sepsis-3 criteria through a posthoc analysis ${ }^{4}$. Patients $<18$ year-old and pregnant women were excluded.

For each patient, the following data were collected:

a) demographic information: age, sex, weight and height;

b) admission to ICU: date, type of admission (medical, surgical in election, emergency surgery), the most common comorbidities (cancer, chronic renal failure, heart failure, diabetes, obesity);

c) diagnosis of abdominal sepsis: diagnostic tools (clinical, abdominal X-ray, abdominal Ultrasound, exploratory laparoscopy, exploratory laparotomy), anatomical integrity (presence or absence of perforation - localized or diffuse peritonitis), diagnosis (primary - secondary - tertiary peritonitis); d) microbiology: cultures with antibiogram either on intra- and postoperative peritoneal fluid (on surgical wound tampons), or on BronchoAlveolar Lavage (BAL), or on urine/ blood cultures, distinguished among multidrug-resistant, extensively drug-resistant and pandrug-resistant bacteria ${ }^{16}$;

e) anti-infective treatment: antimicrobial therapy: drug, daily dose, type of administration (intermittent intravenous, continuous intravenous, oral, intramuscular), duration, type of prescription (empirical or targeted); source control: drainage techniques (percutaneous drainage without surgery, surgical drainage, peritoneal lavage, post-operative drainage), necrotic tissue resection, decompression with laparostomy, reconstruction of normal anatomy; use of additional sepsis therapies: immunoglobulins, hydrocortisone, others;

f) severity of sepsis: in preoperative period (6 hours before surgery), in early post-operative period (24 hours after surgery) and in late post-operative (72 hours after the surgery). The diagnosis was performed evaluating: SOFA score, organ support (mechanical ventilation, vasoactive amines, dialysis), $\mathrm{pH}$, serum lactates, C-reactive protein (CRP), procalcitonin (PCT), white blood cells;

g) fluid balance during the first 5 days of ICU admission;

h) 30-day and 90-day survival.

The primary outcome was 90-day mortality (intermediate term mortality); secondary outcomes were 30-day mortality (short term mortality), duration of antibiotic therapy and duration of hospitalization in ICU.

\section{StATISTICAL ANALYSIS}

The results are reported as mean \pm standard deviation, median [interquartile range], relative risk and 95\% confidence interval, when appropriate. The normality of the distribution was evaluated with Shapiro-Wilk test. The continuous variables were compared by means of the t-test or the Mann-Whitney test, depending on the distribution of data (normal or not normal). Repeated data were processed with ANOVA test for repeated measurements with post-hoc Šidák correction. The categorical variables were compared through the Fisher's exact test. The analysis for repeated measurements was performed using the Kruskal-Wallis test for not normally distributed samples. Values with $p$-value $<0.05$ were considered statistically significant. The predictive performance of each score was assessed with AUC analysis. In accordance with the guidelines, scores were classified as non-predictive (AUC $=0.5$ ), inaccurate $(0.5<\mathrm{AUC}<0.7)$, moderately accurate $(0.7 \leq \mathrm{AUC}<0.9)$, extremely accurate $(0.9 \leq \mathrm{AUC}<1)$ and perfect $(A \cup C=1)$. 


\section{RESULTS}

During the observational study period, thirty patients were enrolled. The clinical and demographic characteristics of the population before and after admission to ICU are summarized in Tables II-IV. Among the patients admitted to ICU with abdominal sepsis, $80 \%$ was aged $\geq 65$ (24 out of 30 ) and $53 \% \geq 75$ years (17 out of 30). Regarding overall mortality, 30-day mortality was $17 \%(n=5)$ and 90-day mortality was $37 \%(n=11)$. All hospitalized patients were affected by pre-existing comorbidities, the most common of which are reported in Table II. Among these, chronic renal failure and diabetes mellitus were more common among patients who died during the follow-up (6/11 vs 1/19 and 2/19, respectively, $p<0.05)$. Analyzing the rate of death in the different age classes, almost all patients who died were $\geq 65$ years old, in particular $27 \%$ were in the middle class of age (between 65 and 75 year) and 64\% in the oldest class ( $\geq 75$ years) (see Figure 1). No other significant difference in mortality was observed at baseline, except for the CRP levels that was directly associated with an increased risk of death $(13 \pm 5$ vs $18.8 \pm 8$, $p=0.03$ ). The postoperative data at 24 hours (Tab. III) and at 72 hours (Tab. IV) confirm an increased mortality linked to CRP values (at 24h: 17.0 [10.7-25.0] vs 26.0 [17.2-32.0], $p=0.01$, at $72 \mathrm{~h}: 16.6 \pm 4.5$ vs $24.0 \pm 8.1$, $p=0.06)$. The CRP trend is shown in Figure 2. No significant differences were found in mortality, in relation

Table II. Characteristics of the population at baseline in relation with the primary outcome.

\begin{tabular}{|l|c|c|c|}
\hline Variables & $\begin{array}{c}\text { Survivor } \\
\mathrm{N}=19\end{array}$ & $\begin{array}{c}\text { Non } \\
\text { survivor } \\
\mathrm{N}=11\end{array}$ & $\mathrm{P}$ \\
\hline Age $\geq 65$ years n,(\%) & $14(73)$ & $10(91)$ & 0.26 \\
\hline Gender (female) n,(\%) & $7(37)$ & $5(45)$ & 0.46 \\
\hline \multicolumn{3}{|c|}{ Comorbidity } \\
\hline Chronic renal disease n,(\%) & $1(5)$ & $6(54)$ & 0.004 \\
\hline Heart failure n,(\%) & $1(5)$ & $1(9)$ & 0.6 \\
\hline Diabetes mellitus n,(\%) & $2(10)$ & $6(54)$ & 0.015 \\
\hline Obesity (BMl > 30) n,(\%) & $5(26)$ & $2(18)$ & 0.48 \\
\hline Neoplasia n,(\%) & $5(26)$ & $2(18)$ & 0.48 \\
\hline MV n,(\%) & $5(26)$ & $4(36)$ & 0.43 \\
\hline Inotropes n,(\%) & $0(0)$ & $1(9)$ & 0.37 \\
\hline qSOFA $\geq 2 \mathrm{n},(\%)$ & $6(31)$ & $5(45)$ & 0.35 \\
\hline Lac (mmol/L) & $2.4 \pm 1.8$ & $2.7 \pm 1,1$ & 0.62 \\
\hline pH & $7.1 \pm 0.3$ & $7.0 \pm 0,1$ & 0.19 \\
\hline CRP (mg/dL) & $13 \pm 5$ & $18.8 \pm 8$ & 0.03 \\
\hline WBC (10\%/L) & $14.7[10-22]$ & $12.6[12-18]$ & 0.61 \\
\hline SOFA score & $7.1 \pm 3$ & $7.55 \pm 3.6$ & 0.77 \\
\hline
\end{tabular}

MV: mechanical ventilation; Lac: serum lactates; CRP: C reactive protein; WBC: white blood cells

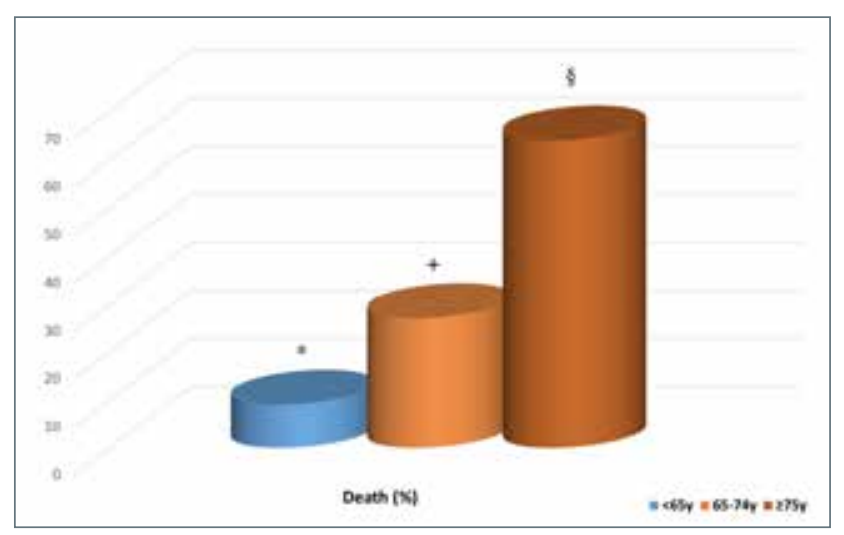

Figure 1. 90-day mortality rate (\%) in relation to age (y). * $p=$ $0.37 ;+p=1 ; \S p=0.47$.

to neither the lactate levels (preoperative: $2.4 \pm 1.8 \mathrm{vs}$ $2.7 \pm 1.1, \mathrm{p}=0.62$; post-operative at $24 \mathrm{~h}: 2.3 \pm 1 \mathrm{vs}$ $2.4 \pm 1.1, \mathrm{p}=0.91$, post-operative at $72 \mathrm{~h}: 1.1 \pm 0.5$ vs $1.62 \pm 0.6, p=0.05$ ) nor the levels of PCT, whose dosage was possible only in the postoperative period (at 24h: 7.0 [1.7-33.0] vs 9.1 [3.5-22.6], $\mathrm{p}=0.14$; a 72h: 6.0 [0.7-15.1] vs 10 [2.8-18.5], $p=0.10)$. In patients with septic shock, blood lactate levels show a trend towards normalization in survivor patients, although without reaching statistical significance $(p=0.11)$.

The most frequent cause of abdominal sepsis was due to secondary peritonitis. In $57 \%$ of total cases, secondary peritonitis was due to bowel perforation or ischemia; the remaining cases were caused by postoperative peritonitis, gangrenous acute appendicitis, biliary tract infections or inflammatory bowel disease exacerbation. The most frequently isolated bacteria in different biological liquids were Escherichia coli (16 isolations), Pseudomonas aeruginosa (14 isolations) and Enterococcus faecium (12 isolations). Pathogens were more frequently isolated in peritoneal fluid (48 total isolations), followed by swabs on surgical wound; 11 isolations were obtained in blood culture.

The only species isolated of fungus was Candida albicans, but less frequently with respect to bacteria: 8 isolations of this fungus were found.

SOFA score was the prognostic score better associated with mortality at $30(A \cup C=0.702)$ and at 90 days $(A \cup C=0.730)$. qSOFA was also somehow predictive of 30-day mortality ( $A \cup C=0.640$ ), but it did not show any

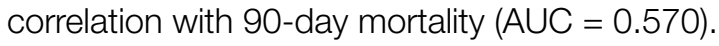

Among patients with positive cultures (70\%), starting an appropriate antibiotic therapy within the first 12 hours from the diagnosis was strongly associated with a reduction in mortality (70 vs $12 \%, p=0.01$ ).

The fluidic balance in patients who survived vs patients who died was comparable in the first 72 hours 
Table III. Patients' characteristics in the early postoperative period (24 h) in relation with the primary outcome.

\begin{tabular}{|l|c|c|c|}
\hline Variables & $\begin{array}{c}\text { Survivor } \\
\mathbf{N}=19\end{array}$ & $\begin{array}{c}\text { Non survivor } \\
\mathbf{N}=11\end{array}$ & P \\
\hline MV $\mathrm{n},(\%)$ & 19 & 11 & 0.99 \\
\hline Inotropes $\mathrm{n},(\%)$ & $5(26)$ & $6(54)$ & 0.46 \\
\hline Lac $(\mathrm{mmol} / \mathrm{L})$ & $2.3 \pm 1$ & $2.4 \pm 1.1$ & 0.91 \\
\hline $\mathrm{pH}$ & $7.35 \pm 0.06$ & $7.33 \pm 0.09$ & 0,58 \\
\hline CRP $(\mathrm{mg} / \mathrm{dL})$ & $17.0[10.7-25.0]$ & $26.0[17.2-32.0]$ & 0.01 \\
\hline PCT $(\mathrm{ng} / \mathrm{mL})$ & $7.0[1.7-33.0]$ & $9.1[3.5-22.6]$ & 0.14 \\
\hline WBC $\left(10^{9} / \mathrm{L}\right)$ & $13.1[11.6-17.3]$ & $12.5[11.8-18.6]$ & 0.94 \\
\hline Dialysis $\mathrm{n},(\%)$ & $6(32)$ & $2(19)$ & \\
\hline
\end{tabular}

MV: mechanical ventilation; Lac: serum lactates; CRP: C reactive protein; PCT: procalcitonin; WBC: white blood cells

Table IV. Patients' characteristics in the late postoperative period (72 h) in relation with the primary outcome.

\begin{tabular}{|l|c|c|c|}
\hline Variables & $\begin{array}{c}\text { Survivor } \\
\mathbf{N}=19\end{array}$ & $\begin{array}{c}\text { Non survivor } \\
\mathbf{N}=11\end{array}$ & P \\
\hline VM $\mathrm{n},(\%)$ & $16(84)$ & $10(91)$ & 0.53 \\
\hline Inotropes $\mathrm{n},(\%)$ & $6(32)$ & $5(45)$ & 0.35 \\
\hline Lac $(\mathrm{mmol} / \mathrm{L})$ & $1.1 \pm 0.5$ & $1.62 \pm 0.6$ & 0.05 \\
\hline $\mathrm{pH}$ & $7.4 \pm 0.04$ & $7.37 \pm 0.09$ & 0.15 \\
\hline CRP $(\mathrm{mg} / \mathrm{dL})$ & $16.6 \pm 4.5$ & $24.0 \pm 8.1$ & 0.06 \\
\hline PCT $(\mathrm{ng} / \mathrm{mL})$ & $6.0[0.7-15.1]$ & $10.0[2.8-18.5]$ & 0.10 \\
\hline WBC $\left(10^{9} / \mathrm{L}\right)$ & $13.6[11.6-17.1]$ & $12.6[11.8-18.3]$ & 0.94 \\
\hline Dialysis $\mathrm{n},(\%)$ & $0(0)$ & $2(18)$ & 0.12 \\
\hline
\end{tabular}

MV: mechanical ventilation; Lac: serum lactates; CRP: C reactive protein; PCT: procalcitonin; WBC: white blood cells

of hospitalization; however, after 72 hours, the fluidic balance was statistically different $(p<0.001)$, resulting higher in patients who died.

\section{DISCUSSION}

The main result of this study is that our data confirm the high prevalence of very old patients admitted to ICU for abdominal sepsis. Moreover, the current study shows the high mortality due to this pathology (37\% of our sample) ${ }^{17}$, especially among older persons: indeed, almost all deaths occurred in patients ${ }^{3} 65$ years, and more than half of patients ${ }^{3} 75$ years died. Therefore, as already reported in the literature ${ }^{3}$, advanced age seems to predict an adverse outcome in patients with abdominal sepsis. Besides, this study demonstrated that SOFA score is associated with short and intermediate term mortality supporting its use as prognostic marker.

The comorbidities can be considered as an additional risk factor for failure to control the infection; in our study, those more correlated to a negative outcome were chronic renal insufficiency ${ }^{18}$ and mellitus diabetes ${ }^{19}$.

Although CRP is recognized as a non-specific index, high CRP levels in septic patients seem correlated to a higher mortality: during the three days of this survey, CRP trend appears to be significantly different between patients who died and not (Fig. 2). Instead, in contrast with the literature ${ }^{20,21}$, neither PCT nor serum lactate levels were related to sepsis severity and mortality. During the first and the third post-operative day, PCT trend shows a lower decrease in patients who died, as

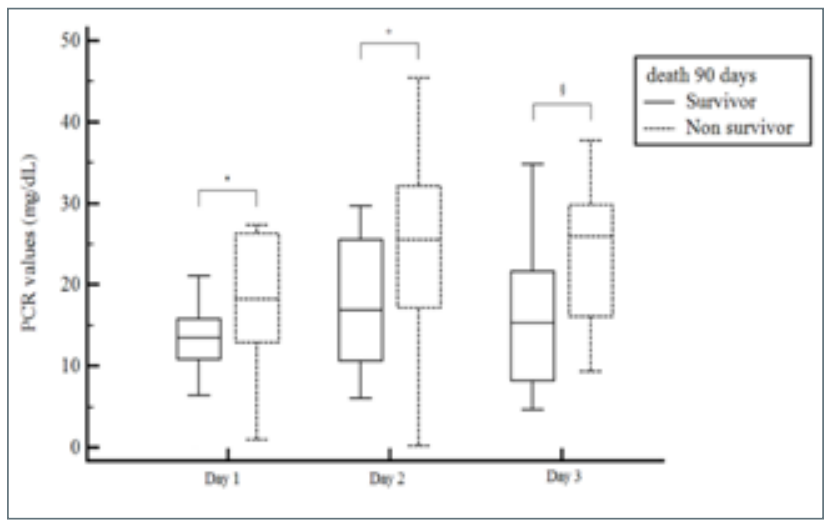

Figure 2. CRP levels $(\mathrm{mg} / \mathrm{dl})$ in the preoperative day (Day 1), in the first (Day 2) and third (Day 3) postoperative day in the recruited population divided in survivor and non-survivor. ${ }^{*} p=$ $0.03 ;+p=0.01 ; \S p=0.06$. 
compared to survivors, although without reaching a statistical significance (Tabs. III-IV). Even serum lactate levels are slightly increased in patients who died, but again without statistical significance (Tabs. II-IV). These results are likely due to the small sample size and the high heterogeneity of patients' characteristics (age, comorbidity, bacteria causing the infection, use of invasive devices); for instance, serum lactate analysis was carried out only on the subgroup of patients with septic shock, further reducing the statistical power of the analysis.

In agreement with previous studies ${ }^{22,23}$, the SOFA score proved a reliable prognostic score of mortality also in geriatric patients, whereas the qSOFA, created on retrospective studies ${ }^{5}$, was related to mortality risk only at 30 days, suggesting that qSOFA needs further validation studies as a prognostic mortality score for sepsis, particularly in the geriatric population.

In accordance with several publications ${ }^{24,25}$, our data demonstrate how an appropriate empirical antibiotic therapy, established in the first 12 hours after diagnosis, leads to a reduction in overall mortality from 70 to $12 \%$ $(p=0.01)$. Thus, a previous hypothesis on the type of bacterium causing the sepsis would be very important, investigating the most common local antibiotic resistances; this procedure highly increases the success probability of the empirical antibiotic therapy with an enormous benefit for the outcome. Among patients with at least one positive culture, 91\% had a polymicrobial infection as etiological cause of abdominal sepsis, recognized by the literature as a highly negative prognostic factor ${ }^{26}$. The most frequently isolated bacteria were $E$. coli, in particular from peritoneal fluid, and $P$. aeruginosa, often found in different biological samples, the latter a clear sign of the higher tendency of Pseudomonas infection to spread out. Enterococci were also very frequent, particularly $E$. faecium.

Moreover, according to the literature ${ }^{3}$, the present investigation confirmed that abdominal sepsis is less frequently caused by fungi; among them, only C. albicans was isolated and it was always sensitive.

In addition to an early and appropriate antibiotic therapy, one of the most important recommendations of Surviving Sepsis Campaign is an aggressive intravenous fluids administration, in order to maintain an arterial pressure sufficient to guarantee adequate tissue perfusion ${ }^{14}$. Typically, in septic patient the fluidic balance is enormously positive, because of the increased permeability of blood capillary wall, especially during the first hours of fluid-therapy. However, as already observed ${ }^{27}$, our results also show an increased mortality in patients with positive fluid balance prolonged over time, in particular over 72 hours: a very positive fluidic balance from the fourth day onwards correlates to an increased mortality in septic patients.
It is worth underlining that qSOFA was created on retrospective studies performed on patients with an median age of 60 years and thus requires a prospective validation on older population for its use in everyday clinical life. The major strength of our study is that this validation is, at least partially, given by the present investigation, a prospective study which analyses qSOFA in a septic population. However, the small sample size (30 patients) with high heterogeneity in clinical characteristics is the main limit of our study. As consequence, this study should be considered as a preliminary report that needs more detailed future investigations to validate the clinical use of qSOFA as prognostic tool in older people with diagnosis of sepsis.

In conclusion, our results confirm the high mortality caused by abdominal sepsis in geriatric patient. Almost all deaths occurred in patients $\geq 65$ years old, underlining as age is a negative prognostic factor during abdominal sepsis. Even in older persons with sepsis, SOFA is a reliable score of mortality, while qSOFA appears to be correlated to 30-days mortality and it could be useful in extra-hospital setting to discriminate patients with an increased risk of death for sepsis.

\section{References}

1 Iwashyna TJ, Cooke CR, Wunsch $\mathrm{H}$, et al. Population burden of long-term survivorship after severe sepsis in older Americans. J Am Geriatr Soc 2012;60:1070-7. https://doi. org/10.1111/j.1532-5415.2012.03989

2 Gaieski DF, Edwards JM, Kallan MJ, et al. Benchmarking the incidence and mortality of severe sepsis in the United States. Crit Care Med 2013;41:1167-74. https://doi. org/10.1097/CCM.0b013e31827c09f8.

3 Martin GS, Mannino DM, Moss M. The effect of age on the development and outcome of adult sepsis. Crit Care Med 2006;34:15-21. https://doi.org/10.1097/01. ccm.0000194535.82812.ba

4 Singer M, Deutschman CS, Seymour CW, et al. The Sepsis definitions Task Force The Third International Consensus Definitions for Sepsis and Septic Shock (Sepsis-3). JAMA 2016; 315:762-74. https://doi.org/10.1001/ jama.2016.0288

5 Levy MM, Fink MP, Marshall JC, et al. SCCM/ESICM/ ACCP/ATS/SIS. 2001 SCCM/ESICM/ACCP/ATS/SIS International Sepsis Definitions Conference. Crit Care Med 2003;31:1250-6. https://doi.org/10.1097/01. CCM.0000050454.01978.3B

6 Vincent JL, Moreno R, Takala J, et al. The SOFA (Sepsisrelated Organ Failure Assessment) score to describe organ dysfunction/failure. On behalf of the Working Group on Sepsis-Related Problems of the European Society of Intensive Care Medicine. Intensive Care Med 1996;22: 707-10. https://doi.org/10.1007/bf01709751

7 Ferreira FL, Bota DP, Bross A, et al. Serial evaluation of 
the SOFA score to predict outcome in critically ill patients. JAMA 2001;286:1754-8. https://doi.org/10.1001/ jama.286.14.1754

8 Karlsson S, Varpula M, RuokonenE, etal. Incidence, treatment, and outcome of severe sepsis in ICU-treated adults in Finland: the Finnsepsis study. Intensive Care Med 2007;33:435-43. https://doi.org/10.1007/s00134-006-0504-z

9 Montravers P, Blot S, Dimopoulos G, et al. Therapeutic management of peritonitis: a comprehensive guide for intensivists. Intensive Care Med 2016;42:1234-47. https:// doi.org/10.1007/s00134-016-4307-6

10 Vincent JL, Rello J, Marshall J, et al. International study of the prevalence and outcomes of infection in intensive care units. JAMA 2009;302:2323-9. https://doi.org/10.1001/ jama.2009.1754

11 Bastoni D, Ticinesi A, Lauretani F, et al. Application of The Sepsis-3 Consensus Criteria in a geriatric acute care unit: a prospective study. J Clin Med 2019;8:359. https://doi. org/10.3390/jcm8030359

12 Tolonen M, Coccolini F, Ansaloni L, et al. Getting the invite list right: a discussion of sepsis severity scoring systems in severe complicated intra-abdominal sepsis and randomized trial inclusion criteria. World J Emerg Surg 2018;13:17. https://doi.org/10.1186/s13017-018-0177-2

13 C Eckmann, M Dryden, P Montravers, et al. Antimicrobial treatment of "complicated" intra-abdominal infections and The New IDSA Guidelines - a commentary and an alternative European approach according to clinical definitions. Eur J Med Res 2011;16:115-26. https://doi. org/10.1186/2047-783x-16-3-115

14 Rhodes A, Evans L, Alhazzani W, et al. Surviving sepsis campaign: international guidelines for management of sepsis and septic shock: 2016. Crit Care Med 2017;45:486-552. https:// doi.org/10.1097/CCM.0000000000002255.

15 Levy MM, Evans LE, Rhodes A. The Surviving Sepsis Campaign Bundle: 2018 Update. Crit Care Med 2018;46:9971000. https://doi.org/10.1097/CCM.0000000000003119.

16 Magiorakos AP, Srinivasan A, Carey RB, e al. Multidrugresistant, extensively drug-resistant and pandrug-resistant bacteria: an international expert proposal for interim standard definitions for acquired resistance. Clin Microbiol Infect 2012;18:268-81. https://doi.org/10.1111/j.14690691.2011.03570.x

17 Leligdowicz A, Dodek PM, Norena M, et al. Association between source of infection and hospital mortality in patients who have septic shock. Am J Respir Crit Care Med 2014;189:1204-13. https://doi.org/10.1164/rccm.201310$18750 \mathrm{C}$
18 Poutsiaka DD, Davidson LE, Kahn KL, et al. Risk factors for death after sepsis in patients immunosuppressed before the onset of sepsis. Scand J Infect Dis 2009;41:46979. https://doi.org/10.1080/00365540902962756.

19 Chirillo F, Bacchion F, Pedrocco A, et al. Infective endocarditis in patients with diabetes mellitus. J Heart Valve Dis 2010;19:312-20.

20 Kip MM, Kusters R, Izerman MJ, et al. A PCT algorithm for discontinuation of antibiotic therapy is a cost-effective way to reduce antibiotic exposure in adult intensive care patients with sepsis. A PCT algorithm for discontinuation of antibiotic therapy is a cost-effective way to reduce antibiotic exposure in adult intensive care patients with sepsis. J Med Econ 2015;18:944-53. https://doi.org/10.3111/13 696998.2015.1064934

21 Casserly B, Phillips GS, Schorr C, et al. Lactate measurements in sepsis-induced tissue hypoperfusion: results from the Surviving Sepsis Campaign database. Crit Care Med 2015;43:567-73. https://doi.org/10.1097/ CCM.0000000000000742

22 Vincent JL, Moreno R, Takala J, et al. The SOFA (Sepsisrelated Organ Failure Assessment) score to describe organ dysfunction/failure. On behalf of the Working Group on Sepsis-Related Problems of the European Society of Intensive Care Medicine. Intensive Care Med 1996;22:70710. https://doi.org/10.1007/bf01709751

23 Ferreira FL, Bota DP, Bross A, et al. Serial evaluation of the SOFA score to predict outcome in critically ill patients. JAMA 2001;286:1754-8. https://doi.org/10.1001/ jama.286.14.1754

24 Ibrahim EH, Sherman G, Ward S, et al. The influence of inadequate antimicrobial treatment of bloodstream infections on patient outcomes in the ICU setting. Chest 2000;118:14655. https://doi.org/10.1378/chest.118.1.146

25 Barie PS, Hydo LJ, Shou J, et al. Influence of antibiotic therapy on mortality of critical surgical illness caused or complicated by infection. Surg Infect 2005;6:41-54. https://doi.org/10.1089/sur.2005.6.41

26 Shorr AF, Tabak YP, Killian AD, et al. Healthcare-associated bloodstream infection: a distinct entity? Insights from a large U.S. database. Crit Care Med 2006;34:2588-95. https://doi.org/10.1097/01.CCM.0000239121.09533.09

27 Boyd JH, Forbes J, Nakada TA, at al. Fluid resuscitation in septic shock: a positive fluid balance and elevated central venous pressure are associated with increased mortality. Crit Care Med 2011;39:259-65. https://doi.org/10.1097/ CCM.0b013e3181feeb15 